\title{
DynaMo - Dynamic 3D Models for the Web
}

\section{A declarative approach to dynamic and interactive 3D models on the web using $\mathrm{x} 3 \mathrm{dom}$}

\author{
Ulrich Krispel ${ }^{*}$ \\ Institute for Computer Graphics and \\ Knowledge Visualization (CGV), \\ Graz University of Technology \\ Graz, Austria \\ Fraunhofer Austria Research GmbH \\ Graz, Austria \\ ulrich.krispel@fraunhofer.at
}

\author{
Volker Settgast \\ Institute for Computer Graphics and \\ Knowledge Visualization (CGV), \\ Graz University of Technology \\ Graz, Austria \\ Fraunhofer Austria Research $\mathrm{GmbH}$ \\ Graz, Austria \\ volker.settgast@fraunhofer.at
}

\author{
Dieter W. Fellner \\ Institute for Computer Graphics and \\ Knowledge Visualization (CGV), \\ Graz University of Technology \\ Graz, Austria \\ GRIS, TU Darmstadt \& \\ Fraunhofer IGD \\ Darmstadt, Germany
}

\begin{abstract}
Animations aid greatly to a presentation of a sophisticated manmade object. With additional interactivity, a user can explore such an object to gain even better understanding. The authoring of such dynamic models is often very resource demanding, as the animations and logic of interaction has to be expressed in an authoring program; often using a programming language. Furthermore, the 3D model and its dynamic capabilites are tightly coupled, which makes it costly to integrate changes of the underlying 3D model e.g. if a machine part changes.

Contribution and Benefit. We present a novel scheme to define varying states of a 3D model in a decoupled, declarative manner using pattern matching. Furthermore, we demonstrate its capabilites for the web with an open source JavaScript implementation that operates on an x3dom model.
\end{abstract}

\section{CCS CONCEPTS}

- Information systems $\rightarrow$ Web applications; • Computing methodologies $\rightarrow$ Computer graphics; Procedural animation;

\section{KEYWORDS}

animation, x3dom, declarative

\section{ACM Reference Format:}

Ulrich Krispel, Volker Settgast, and Dieter W. Fellner. 2018. DynaMo - Dynamic 3D Models for the Web: A declarative approach to dynamic and interactive 3D models on the web using x3dom. In Web3D '18: The 23rd International Conference on Web3D Technology, fune 20-22, 2018, Poznan, Poland. ACM, New York, NY, USA, 5 pages. https://doi.org/10.1145/3208806.3208812

\footnotetext{
${ }^{*}$ Corresponding Author
}

Permission to make digital or hard copies of part or all of this work for personal or classroom use is granted without fee provided that copies are not made or distributed for profit or commercial advantage and that copies bear this notice and the full citation on the first page. Copyrights for third-party components of this work must be honored. For all other uses, contact the owner/author(s).

Web3D '18, fune 20-22, 2018, Poznan, Poland

(C) 2018 Copyright held by the owner/author(s)

ACM ISBN 978-1-4503-5800-2/18/06.

https://doi.org/10.1145/3208806.3208812

\section{INTRODUCTION}

As computers and devices are becoming more powerful with less power consumption, web applications are getting richer and graphically elaborate, often with 3D content. Furthermore, since the advent of the WebVR specification, virtual reality (VR) applications can be experienced in the web browser. Due to their realistic representation, 3D models are a favorable way of illustration, especially in the sales and presentation domain. Furthermore, the presentation or understanding of a scene is even better when the scene is dynamic, i.e. the 3D model varies over time - such as a moving or rotating part of a machine - even more so if the model becomes interactive.

The presentation in the web is only one step in the development of a product, the planning and modeling is done in earlier stages. Product Lifecycle Management (PLM) systems integrate the digital representation of the product development. The web presentation should therefore reuse the information present in PLM systems.

The authoring of dynamic 3D scenes in the browser is often time consuming, as the scene needs to be transformed in a format that is suited for display in the browser; dynamic capabilites and interaction logic needs to be scripted or defined using an authoring system. The resulting web application typically induces a tight coupling between animation and interaction and the 3D scene. There is evidence that tight coupling of code components increases technical debt [MacCormack and Sturtevant 2016]; thus, a tight coupling of 3D scene and dynamic capabilities may introduce elaborate workflows, especially if the model changes, for example when a new variant of a model should be introduced in a web shop; another example is that a model should have several geometric variants when the structure does not change, e.g. a low-poly version for a VR headset with weak display capacity.

Therefore, we propose to model the dynamic capabilities of a 3D scene as a separate declarative description with an accompanying library that simplifies the dynamic modeling process, an overview of the method can be seen in Figure 1. We demonstrate the method by providing an open source implementation that operates on x3dom [Behr et al. 2009], the open and human-readable 3D scenegraph embedded in the HTML DOM which is based on the X3D standard [ISO 19775-1 2013]. 

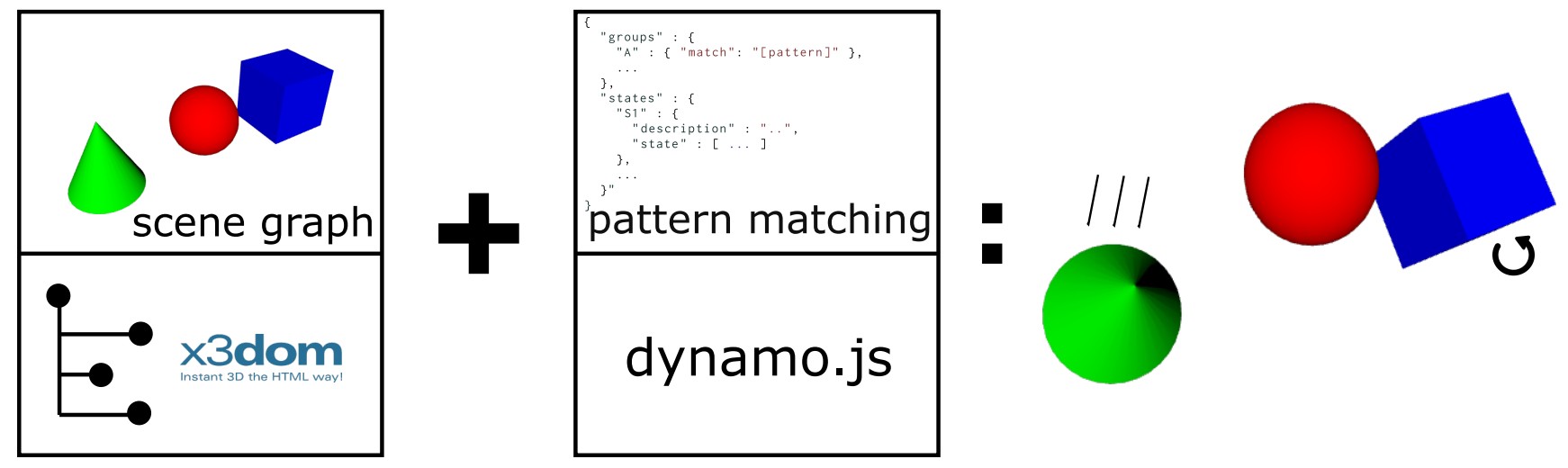

Figure 1: A static scene, represented as a hierarchical $\mathbf{x} 3 \mathrm{~d} / \mathbf{x} 3 \mathrm{dom}$ model, is augmented with dynamic changes, described as a declarative description of states using JSON, and a thin application layer that represents the interactive logic. The dynamo.js libary performs state application and visual transitioning (animation) between states. The separation of 3D model and dynamic properties enables better authoring loops due to an easy replacement or outside modification of the underlying $3 \mathrm{D}$ scene.

\section{RELATED WORK}

The classical approach to dynamic content creation stems from traditional hand-drawn animation: a set of key frames is associated to specific points in the timeline, and intermediate frames are generated between two key frames, which is called inbetweening, or tweening [Kort 2002].

The proposed system builds on the aforementioned technique of key framing and the idea of representing a dynamic system using a graph structure, as is common in the area of character animation: graph based motion synthesis [Arikan and Forsyth 2002], [Kovar et al. 2002], [Lee et al. 2002]. Similar to such systems we allow a set of arbitrary states and transitions between them. Additionally, the system uses pattern matching to identify scene graph nodes to which the animation will be applied, similar to Cascading Style Sheets (CSS). While x3dom [Behr et al. 2009] and other methods [Seo et al. 2017] utilize CSS to define 3D transformations, it does not allow the aggregation of several animations at once to the same attribute of a Document Object Model (DOM) element.

Our solution was motivated by a practical example: creating an interactive model of an existing representation from a PLM system. The industry standard for exchange data of such systems is the ISO STEP Standard which stands for "Standard for the Exchange of Product model data" [ISO 10303-242 2014]. We utilized pythonOCC, a python binding to the OpenCascade modeling kernel, to convert the STEP representation to X3D [Paviot 2018].

There exists a large amount of software that allows to author interactive scenes, e.g. game engines that also deploy to the web browser, such as Unity3D [Unity Technologies 2018]; Blend4Web [Blend4web 2018] is a plug-in for the open source modeling suite Blender [Foundation 2018] that allows to export interactive 3D content to the web browser. To our knowledge the use of such engines always introduces tight coupling between application logic and the 3D scene.

Thus, we build upon the open x3dom system to represent dynamic scenes in the web. x3dom also offers dynamic capabilites [Behr et al. 2011]: dynamic content can be added to the scene graph using time sensors, routes and interpolation nodes to modify content.
There exist also domain-specific extensions to $\mathrm{x} 3 \mathrm{~d} / \mathrm{x} 3 \mathrm{dom}$, utilizing a more abstract scene definition, for example the extensions for animation control of humanoid modeling [Jung and Behr 2008]. Our declarative dynamic description is not intended to replace these mechanisms on implementation level, but to serve as a more convenient and decoupled description of dynamic content - especially for authoring.

\section{DYNAMIC MODELS}

We define a dynamic model as a 3D model that is composed of several parts with dynamically varying properties, e.g. transformation or color. In Computer Aided Engineering (CAE) and Computer Aided Design (CAD), the abstract representation of a complex model consists of a tree or forest-like data structure that models hierarchical relationships from the whole model down to the parts that the model is composed of, e.g. bolts and screws.

Systems for the visualization of dynamic content also often use a hierarchical representation of a scene called the scene graph, that represents the hierarchy of a dynamic scene. The leaves of such a scene graph contain visible elements that represent the smallest possible unit of change of the scene. The scene can be visibly changed by modifying the scene graph and its attributes, e.g. the location of a node. We require a dynamic model to provide support for the following dynamic properties: define varying state information and the ability to perform transitions from one state to an other. State information corresponds to specific properties of a 3D model, e.g. the position of a part. Transitions, i.e. creating several intermediate appearances of the 3D model between states, should be handled automatically.

\subsection{A Declarative Description of Model State}

As described before, the structure of a 3D model corresponds to a tree like structure. The state we want to describe or modify corresponds to attribute values of nodes in this hierarchy. Thus, to describe the state we need to identify the corresponding node(s) and assign values to the attributes. We assume that the input model contains unique labels or other distinguishable meta data for distinct 

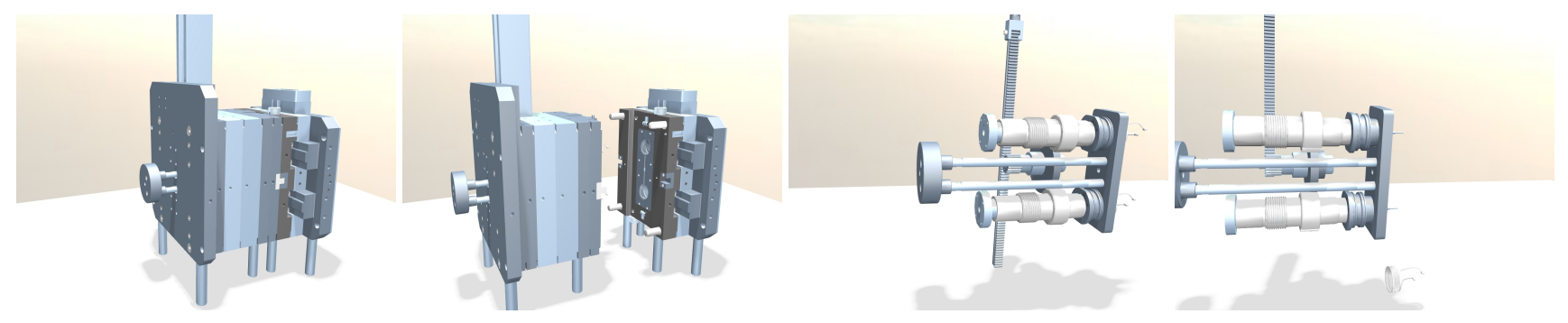

Figure 2: The system was used to animate parts of a complex model that consists of 499 single parts. Specifying the dynamic behavior using a high level description reduces the complexity to define several animation states using only a handful of matching groups. The animation can be shown using the complete model (left) and only specific parts of the inside that demonstrate the inner workings (right).

parts, as is common in CAD models. We do not want a dependency on the concrete structure, or hierarchy of the CAD model. Thus, we propose to use pattern matching on node attributes, e.g. the label, to select desired nodes for state definition. A state definition consists of the attribute name, e.g. translation, and its state value. The value is seen as relative or incremental to any initial state of the attribute in the model. We now group several such match-attribute-value assignments together as a named entity which corresponds to one named state of the dynamic model.

\subsection{State Value Aggregation}

To determine the composite value of each attribute of a named state, all value assignments for one attribute need to be aggregated, additionally, an initial value assignment may be defined in the model. Currently, we use a simple heuristic for state value aggregation, dependent on the type of attribute. Translations are simply added, rotations are combined using consecutive application with quaternions, for visibility we chose that show assignments override hide assignments, etc.

\subsection{State Transitions and Animations}

In order to animate a model we let the system perform automatic transitions between the states defined in the previous subsection. If the system performs a transition from state $A$ to state $B$, it interpolates between the aggregated attribute values of $A$ and $B$. The value is used for the transition as a constant when it is defined in $A$ only, or a transition between the current model value of that attribute to the definition is made when it is defined in $B$ only.

For a state transition, the values relevant attribute consist of booleans or various numerical value types: scalars and vectors. Boolean values or discrete values are switched after half of the transition time, numerical values are linearly interpolated for the transition. Optionally, easing curves may be used to create a visually more appealing transition [Dragicevic et al. 2011]. We define an animation to be a (possibly repeating) sequence of arbitrary state transitions. This also implies that distinct animations may share the same states. The graph of possible transitions is implicitly encoded in the application layer, which handles all interaction logic. As all state definitions are defined to be incremental, the system also allows to apply several transitions between two states at once, as long as these transitions do not interfere with each other.

\section{DYNAMO.JS: AN X3DOM BASED IMPLEMENTATION}

We present an implementation of the dynamic model concept described in Section 3 in the browser, building upon JavaScript and x3dom [Behr et al. 2009]. The static model is encoded as an x3dom scene, as $\mathrm{x} 3 \mathrm{dom}$ also allows to include $\mathrm{X} 3 \mathrm{D}$ files using an inline node, any X3D model may be used as well.

We first define the declarative description of the dynamic capabilites to be a JavaScript Object Notation (JSON) object, called the DynaSpec, as shown in Listing 1 of the appendix. The specification of a dynamic model consists of a list of pattern matching groups that identify parts of the scene, and a number of named states that consist of a list of attribute-value assignments for scene graph nodes or pattern match node groups. Animations are sequences of state transitions. The implementation is a small JavaScript library called dynamo.js, it defines the DynamoInstance class, each instance of this class is bound to an x3dom canvas, and operates on the $\mathrm{x} 3 \mathrm{dom}$ scene of this canvas. The core functionality of the DynamoInstance is built upon the following interface:

constructor upon initialization, the DynamoInstance object is bound to a specific $\mathrm{x} 3 \mathrm{dom}$ canvas on which it will perform dynamic state application.

setState this method immediatly applies all state information that has been associated to the named state that is given as parameter

doStateTransition this method immediatly performs a transition between two given named states, in the specified amount of time

enable/disableAnimation enables or disables the specified animation

\subsection{Implementation Notes}

For efficiency reasons, the system performs the group matching in a preprocessing step. It traverses the scene graph and collects matching nodes, and initial attribute values for assignments. A node matches if the corresponding DOM node id or X3D DEF name matches the given regular expression; alternatively, an arbitrary attribute can be specified for matching.

After all matching nodes of all groups have been collected, the aggregated attribute values are precalculated for each state. Thus, transition values between states can be calculated efficiently from 
these pre-aggregated values. The system allows for enabling or disabling animations, which may be one-shot or cyclic, and launch arbitrary transitions between two states or enable/set any desired state definition using the dynamo. js API. The system also allows for asynchronous event registration by registering callbacks for state change or transition finishing events.

\subsection{A Real World Example}

The proposed system was motivated by a task that involved the presentation and description of a complex machine that creates plastic parts using injection moulding. The 3D model of the machine was given as a STEP export; it consists of 581 nodes, and a total of 499 single shapes instances, of which 186 are distinct base shapes. On the top level, it consists of 62 composite parts that are used to define dynamic behavior.

The CAD model was converted to X3D using pythonOCC, the application was written as an $\mathrm{x} 3 \mathrm{dom}$ html file that includes the X3D model using an inline node. The animation of the machine involves moving two groups of parts apart from each other, and a cogwheel mechanism to move internal parts of the machine. Furthermore, the interactive model should provide a mechanism to hide outer machine parts, so the cogwheel mechanism can be inspected. Using the proposed system, we modeled this behavior using an animation that models the machine functionality with 6 states, and define two additional states that model the visibility state of showing all parts or internal parts. The total dynaspec file consists of 150 lines of JSON, 6 groups, 1 animation and 8 states.

\section{DISCUSSION AND FUTURE WORK}

We propose a method for defining dynamic behavior of a 3D scene using human readable, declarative specification that is decoupled from that scene; we show first results for the web using an implementation that is based on x3dom.

In contrast to developing animations natively in x3dom using time sensors, interpolators and routes, the proposed description describes such behaviour on a higher level and is therefore more convenient to use in an authoring phase where parts of the dynamic behavior naturally changes during the development. Decoupling the declarative specification of dynamic behavior from the implementation also allows to exchange the underlying implementation. For example, the current JavaScript implementation periodically tracks and updates dynamic node attributes. This was sufficient for the larger model described in Section 4.2; if necessary in the future, the implementation could be rewritten to use the existing x3dom dynamic mechanics, such as routes and interpolators to support even larger models. We see great potential in extending the functionality to incorporate different methods of aggregation for state values if the necessity arises, as well as better explicit animation graph representation. Furthermore, a method for automatic placement of labels [Götzelmann et al. 2005], [Stein and Décoret 2008] would also aid visual exploration.

In a broader outlook, we picture the methodology to be able to describe dynamic behavior with respect to all kinds of CAD models, and have different deploy targets such as 3D modeling suites as blender, or game engines, and the web.
Some examples and the dynamo. js library are publicly available under https://github.com/FhA-VC/DynaMo.

\section{ACKNOWLEDGMENTS}

This work was supported by the regional government of Styria, Austria under grant no. GZ: ABT08-139380/2017-11, and the Austrian Research Promotion Agency (FFG) under the Innovationsscheck program. The CAD data model serving as data foundation for the model shown in Figure 2 was kindly provided by Lercher Werkzeugbau $\mathrm{GmbH}$, Austria.

\section{REFERENCES}

Okan Arikan and David A. Forsyth. 2002. Interactive Motion Generation from Examples. ACM Trans. Graph. 21, 3 (July 2002), 483-490. https://doi.org/10.1145/566654.566606

Johannes Behr, Peter Eschler, Yvonne Jung, and Michael Zöllner. 2009. X3DOM: A DOM-based HTML5/X3D Integration Model. In Proceedings of the 14th International Conference on 3D Web Technology (Web3D '09). ACM, New York, NY, USA, 127-135. https://doi.org/10.1145/1559764.1559784

Johannes Behr, Yvonne Jung, Timm Drevensek, and Andreas Aderhold. 2011. Dynamic and Interactive Aspects of X3DOM. In Proceedings of the 16th International Conference on 3D Web Technology (Web3D '11). ACM, New York, NY, USA, 81-87. https://doi.org/10.1145/2010425.2010440

Blend4web. 2018. blend4web - Unleashing the Power of 3D Internet. (2018). Retrieved February 13, 2018 from https://www.blend4web.com/en/

Pierre Dragicevic, Anastasia Bezerianos, Waqas Javed, Niklas Elmqvist, and Jean-Daniel Fekete. 2011. Temporal Distortion for Animated Transitions. In Proceedings of the SIGCHI Conference on Human Factors in Computing Systems (CHI '11). ACM, New York, NY, USA, 2009-2018. https://doi.org/10.1145/1978942.1979233

Blender Foundation. 2018. blender.org - Home of the Blender project - Free and Open 3D Creation Software. (2018). Retrieved February 13, 2018 from https: //www.blender.org/

Timo Götzelmann, Kamran Ali, Knut Hartmann, and Thomas Strothotte. 2005. Form Follows Function: Aesthetic Interactive Labels. In Proceedings of the First Eurographics Conference on Computational Aesthetics in Graphics, Visualization and Imaging (Computational Aesthetics'05). Eurographics Association, Aire-laVille, Switzerland, Switzerland, 193-200. https://doi.org/10.2312/COMPAESTH/ COMPAESTH05/193-200

ISO 10303-242. 2014. Industrial automation systems and integration - Product data representation and exchange - Part 242: Application protocol: Managed model-based $3 D$ engineering. Standard. International Organization for Standardization, Industrial data, Geneva, $\mathrm{CH}$

ISO 19775-1. 2013. Extensible 3D (X3D) - Part 1: Architecture and base components. Standard. International Organization for Standardization, Computer graphics, image processing and environmental data representation, Geneva, $\mathrm{CH}$.

Yvonne Jung and Johannes Behr. 2008. Extending H-anim and X3D for Advanced Animation Control. In Proceedings of the 13th International Symposium on $3 D$ Web Technology (Web3D '08). ACM, New York, NY, USA, 57-65. https://doi.org/10.1145/ 1394209.1394224

Alexander Kort. 2002. Computer Aided Inbetweening. In Proceedings of the 2Nd International Symposium on Non-photorealistic Animation and Rendering (NPAR '02). ACM, New York, NY, USA, 125-132. https://doi.org/10.1145/508530.508552

Lucas Kovar, Michael Gleicher, and Frédéric Pighin. 2002. Motion Graphs. ACM Trans. Graph. 21, 3 (July 2002), 473-482. https://doi.org/10.1145/566654.566605

Jehee Lee, Jinxiang Chai, Paul S. A. Reitsma, Jessica K. Hodgins, and Nancy S. Pollard. 2002. Interactive Control of Avatars Animated with Human Motion Data. ACM Trans. Graph. 21, 3 (July 2002), 491-500. https://doi.org/10.1145/566654.566607

Alan MacCormack and Daniel J. Sturtevant. 2016. Technical debt and system architecture: The impact of coupling on defect-related activity. Fournal of Systems and Software 120 (2016), 170 - 182. https://doi.org/10.1016/j.jss.2016.06.007

Thomas Paviot. 2018. pythonOCC, 3D CAD/CAE/PLM development framework for the Python programming language. (2018). Retrieved February 13, 2018 from http://www.pythonocc.org/

Daeil Seo, Byounghyun Yoo, and Heedong Ko. 2017. Webized 3D Content Streaming System for Autostereoscopic 3D Displays. In Proceedings of the 22Nd International Conference on $3 D$ Web Technology (Web3D '17). ACM, New York, NY, USA, Article 6, 6 pages. https://doi.org/10.1145/3055624.3075940

Thierry Stein and Xavier Décoret. 2008. Dynamic Label Placement for Improved Interactive Exploration. In Proceedings of the $6^{\text {th }}$ International Symposium on Nonphotorealistic Animation and Rendering (NPAR '08). ACM, New York, NY, USA, 15-21. https://doi.org/10.1145/1377980.1377986

Unity Technologies. 2018. Unity 3D. (2018). Retrieved February 13, 2018 from https://unity3d.com/ 


\section{APPENDIX: DYNAMIC MODEL EXAMPLE}

This tutorial consists of a simple but complete example scene with three objects and two example states, to demonstrate the simplicity of the approach. The example can be run in any browser that fulfills the x3dom prerequisites. This example can be directly evaluated using the developer tools that are integrated in common web browsers. See this example html file that defines a simple scene:

\section{Listing 1: Example x3dom scene}

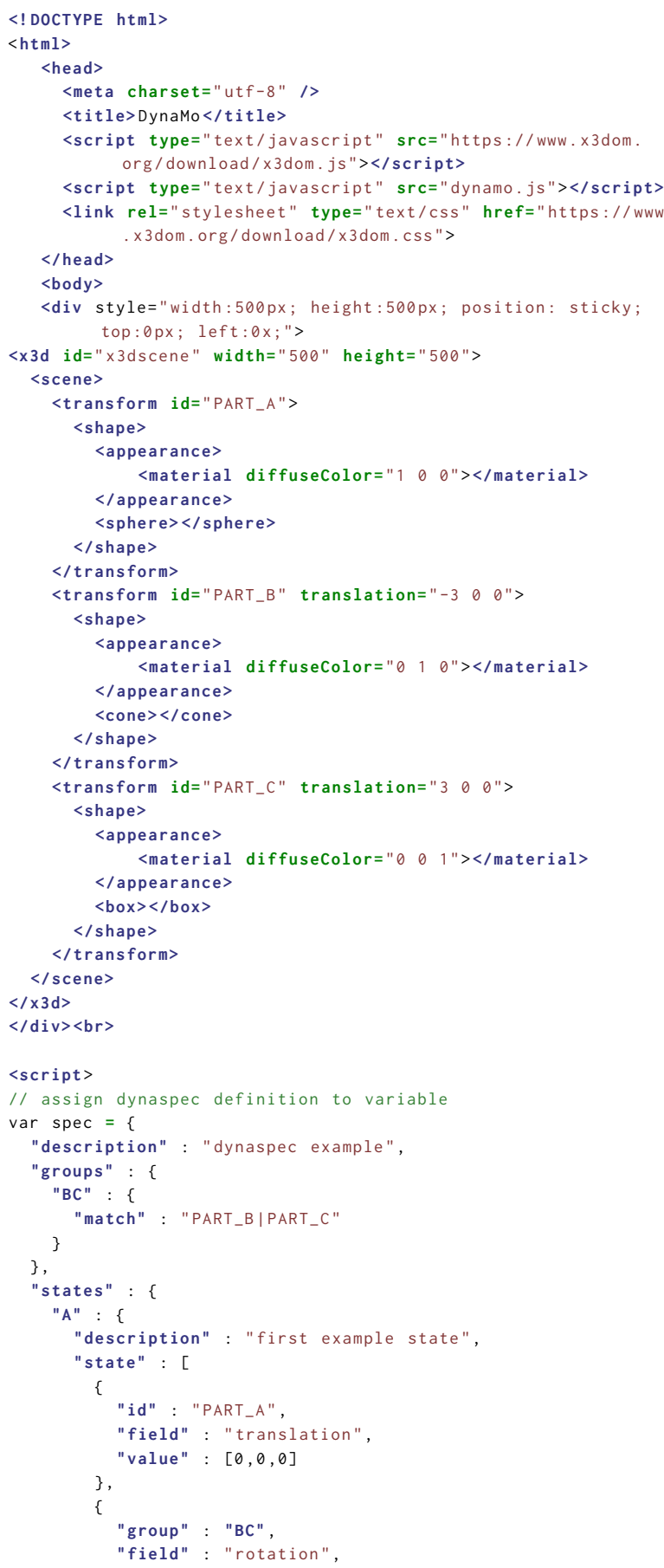

